\title{
Contribución de la aceptación-rechazo materno y paterno al ajuste psicológico de los hijos: Diferencias entre población clínica y general
}

\author{
Mercedes Rodríguez ${ }^{1,2}$, Miguel Á. Carrasco ${ }^{1}$, Francisco P. Holgado-Tello ${ }^{1}$ \\ ${ }^{1}$ Universidad Nacional de Educación a Distancia (UNED), Madrid, España \\ ${ }^{2}$ Escuela Vasco Navarra de Terapia Familiar, Bilbao, España
}

Resumen: La aceptación-rechazo parental se ha revelado como un importante factor de riesgo para el ajuste psicológico de los hijos y su adaptación. El objetivo: Analizar la capacidad predictiva del comportamiento parental percibido por los hijos sobre su pertenencia a un grupo clínico vs otro general. Se analizaron la aceptacion-rechazo materno y paterno y la implicación paterna (expresiva e instrumental) considerando el sexo de los hijos. La muestra estuvo integrada por 226 sujetos (61.94 \% varones) con una media de edad de 14.08 años: 113 de una muestra incidental clínica y 113 de la población general. Resultados: El rechazo indiferenciado de ambos padres fue la variable que mejor predecía la pertenencia de los hijos al grupo clínico. El rechazo paterno vs. materno tendía a resultar más predictivo de la condición clínica, los elementos cualitativos prevalecieron sobre los cuantitativos relativos a la mera participación de los padres en la vida de los hijos.

Palabras clave: Aceptación-rechazo parental; implicación del padre; discriminante; ajuste psicológico.

Contribution of maternal and paternal acceptance-rejection to the children's psychological adjustment: Clinical versus general population

\begin{abstract}
Parental acceptance-rejection has emerged as an important risk factor for a child's psychological adjustment. The aim was to analyze the predictive capacity of parental behavior as perceived by children inasmuch as belonging to a clinical or a general group of participants. The perceived maternal and paternal acceptance-rejection, and the perceived parental involvement (expressive and instrumental) considering the sex of children were analyzed. The sample consisted of 226 subjects (61.94\% boys) with a mean age of 14.8 years: 113 came from an incidental clinical sample and 113 from the general population. The variable to better predict belonging to the clinical group versus the general group was undifferentiated rejection by both parents. However, the perceived paternal rejection was a better predictor of the clinical condition than the maternal rejection condition, with qualitative components of parent-child relations prevailing over quantitative ones relative to the mere participation of the parents in the life of children.
\end{abstract}

Keywords: Parental acceptance-rejection; father involvement; discriminant; psychological adjustment.

\section{Introducción}

Numerosas investigaciones muestran que la mayoría de las personas con alteraciones mentales experimentaron sus

Recibido: 26 febrero 2016; aceptado 21 de junio de 2016.

Correspondencia: Mercedes Rodríguez, Facultad de Psicología, Universidad Nacional de Educación a Distancia, Juan del Rosal, 10, 28040 Madrid, España. Correo-e: rrmertxe@gmail.com

Agradecimientos: Este trabajo ha sido subvencionado por el Ministerio de Ciencia e Innovación del Gobierno de España como parte del proyecto I+D+I Ref. PSI2011-28925. primeros síntomas antes de los 24 años. Pese a que los jóvenes según recoge la OMS, son considerados un grupo de edad sano, un $20 \%$ sufren algún tipo de alteración psicológica entre las que destacan diferentes problemáticas como depresión, ansiedad, abuso de sustancias, comportamiento disruptivo o agresivo, déficit de atención y problemas de hiperactividad y trastorno de estrés postraumático. En este contexto, la familia y las interacciones que en ella se producen, constituye una referencia inexcusable para cualquier intento de aproximación a los problemas de ajuste psicológico en los hijos.

En línea con estas premisas, una de las aproximaciones teóricas que han explorado transculturalmente las re- 
laciones familiares con el ajuste infantil, ha sido la Teoría de aceptación-rechazo parental PARTheory (Parental acceptance-rejection theory) (Rohner, Khaleque, y Cournoyer, 2012). Actualmente revisada y replanteada como la Teoría de aceptación-rechazo interpersonal (IPARTheory) (Rohner, 2014). Desde esta aproximación se intenta predecir y explicar las consecuencias que la percepción de la aceptación-rechazo interpersonal tiene sobre los niveles de ajuste psicológico y de la personalidad. En el contexto familiar, cuando los hijos no satisfacen esta necesidad emocional de afecto, entendida como biológica y permanente, por parte de sus figuras parentales tienden a responder con un patrón específico de respuestas emocionales y conductuales desajustadas (p.ej., hostilidad, agresión, falta de respuesta emocional, baja autoestima y autoeficacia, percepción negativa del mundo y dependencia inmadura o defensiva) que puede llegar a manifestarse con diversas alteraciones interiorizadas o exteriorizadas tales como depresión, conducta suicida, ansiedad, agresividad, hostilidad y delincuencia (Ali, Khaleque y Rohner, 2015; Khaleque, 2015; Khaleque y Rohner, 2012; Rohner et. al., 2012).

En la mayoría de los trabajos realizados desde esta aproximación se ha analizado tanto el papel de la aceptación-rechazo materno como paterno en el ajuste psicológico de los hijos y la conclusión más contundente es que, al menos, son tan importantes uno como el otro (Ali et al., 2015). Aunque, aún es controvertido establecer la contribución diferencial del padre y la madre al ajuste psicológico de los hijos resulta indiscutible el papel de la implicación y participacion del padre bien directa $\mathrm{o}$ indirectamente (p.ej., porporcionando el sustento familiar, apoyando a la madre) (Goodman, 2014) en el desarrollo cognitivo y el ajuste conductual y emocional de los hijos (Flouri, Midouhas, $y$ Narayanan, 2015). Como han mostrado más de cuatro décadas de investigación, la implicación y el amor paternos son realmente importantes para la adaptación de los hijos, y no sólo el amor materno puede postularse como factor exclusivo o suficiente para garantizar el desarrollo psicosocial saludable en los hijos (Lamb, 2012). Es importante considerar que las relaciones de aceptación-rechazo parental y el ajuste psicológico en los hijos ha encontrado evidencia bidireccional (Carrasco, Holgado, Rodríguez y del Barrio, 2009; Hale, VanderValk, Akse, y Meeus, 2008) y no sólo en la dirección del efecto del comportamiento parental sobre los hijos, no obstante la mayoría de los trabajos analizados han explorado las consecuencias unidireccionales del rechazo parental sobre la salud de los hijos.

En muchos trabajos, sin embargo, el papel del padre se ha analizado desde una perspectiva más cuantitativa (tiempo e implicación en actividades y tareas) que cualita- tiva (relación de aceptación-rechazo percibida por los hijos) lo que deja sin aclarar si es la relevancia de la mera implicación paterna en las actividades cotidianas de los hijos o la cualidad de las relaciones padre-hijos lo que realmente contribuye a su ajuste psicológico (para una revisión véase Lamb, 2012; Pleck, 2010)

Más allá del indudable papel de la figura paterna en el ajuste y desarrollo de los hijos, algunos trabajos de metanálisis han mostrado que la aceptación-rechazo paterna percibido por los hijos tiende a contribuir de manera más robusta a su ajuste psicológico que la aceptación-rechazo materna (Khaleque y Rohner, 2012). Estos resultados no siempre se han confirmado y existen estudios que desde otras perspectivas resaltan el papel del afecto materno sobre el paterno (Dwairy, 2010). Lo que parece claro es que más estudios son necesarios para abordar esta controversia entre la relevancia del padre y la madre, y particularmente en relación con el carácter clínico o no de la sintomatología de los hijos. La mayoría de los trabajos han analizado estas diferencias con población general y la significación clínica de los síntomas en los hijos es un indicador de gravedad que sirve de contraste para profundizar en el papel relevante y diferencial que la aceptación materna y paterna poseen respecto a la sintomatología manifiesta en la población general. No se puede obviar, que el rechazo de los padres se ha evidenciado como un factor significativo en la salud mental de los hijos y sus alteraciones psicopatológicas (Dwairy, 2010). Prueba de ello, son los trabajos que han encontrado que los jóvenes pertenecientes a grupos clínicos perciben mayor rechazo y menor cariño de sus padres, lo que ha sido evidenciado en diferentes alteraciones tales como el trastorno obsesivo compulsivo, los trastornos de conducta o la agresividad, la depresión, el maltrato, problemas de somatización y colon irritable (Kostic, Nešić, Stankovic, y Žikić, 2014; Malik, 2012; Najam y Kausar, 2012; Xing, Hou, Zhou, Qin y Pan, 2014). En algunas de estas alteraciones el rechazo de ambos padres se ha constatado significativo (Malik, 2012; Xing et al., 2014) pero en otras prevalecía el rechazo materno (Kostic et al., 2014) y en otras el paterno (Bisht et al., 2008).

Las relaciones entre padres o madres e hijos han mostrado diferencias en función del sexo de los mismos, lo que requiere analizar las variables de aceptación parental y ajuste psicológico considerando esta variable. Los resultados al respecto se han mostrado algo dispares cuando se considera la crianza materna y paterna por separado Algunos estudios muestran que las madres y los padres exhiben la misma sensibilidad en la atención a sus hijos, implicación, comportamiento o control (Asbourne, Daly y Brown, 2011) pero otros muestran diferencias (Kwon, Jeon, Lewsader, y Elicker, 2012). En ocasiones, han en- 
contrado que los padres están más involucrados con sus hijos varones que con sus hijas (Najam y Kausar, 2012) y prefieren interactuar con aquellos. Otros, en cambio, muestran que los padres están más vinculados con su hijas (Demidenko, Manion, y Lee, 2015), mientras que otros no encuentran diferencias. A pesar de estas diferencias en el comportamiento parental en hijos e hijas, lo que un reciente meta-análisis ha revelado es que la contribución de la aceptación parental al ajuste psicológico de los hijos no está moderada por el sexo de estos, y por tanto, la aceptación-rechazo materno o paterno se asocia de forma similar en chicos y chicas (Ali et al., 2015). Todo ello pone de manifiesto la falta de conclusión sobre este tema y la necesidad de considerar la variable sexo de los hijos en el análisis de la conducta parental.

Considerando los trabajos previos realizados, el objetivo de esta investigación es analizar el valor predictivo que tienen la implicación del padre y las dimensiones de aceptación-rechazo paternas y maternas percibidas por los hijos sobre la pertenencia de los niños a una muestra general o clínica. Además se analizará el sexo de los hijos como potencial variable moderadora. Entre las dimensiones parentales se incluyen dimensiones cualitativas (p.ej., aceptación-rechazo percibidos) y en el caso particular de la figura paterna se incluyen además de éstas, dimensiones de carácter más cuantitativo (p.ej., implicación). Dado el menor número de estudios que han investigado la implicación del padre, en el presente trabajo se evaluará específicamente esta variable en la figura del padre y no de la madre. En las culturas patriarcales, como es la cultura española, la implicación materna es un hecho altamente constatado que tiende a aparecer por defecto en las familias y cuya contribución es ya conocida.Todo ello permitirá indagar sobre la relevancia del comportamiento parental, específicamente de la implicación paterna, en la pertenencia de los hijos a un grupo clínico cuya sintomatología ha requerido la atención por parte de los Servicios de salud mental.

\section{Método}

\section{Muestra}

La muestra total está integrada por 226 sujetos procedentes de dos grupos equivalentes: 113 proceden de una población clínica y los otros 113 de la población general o normativa obtenida en centros escolares. Ambos grupos se encuentran equilibrados en cuanto a las variables sexo (61.94\% varones), edad en el caso de las chicas y chicos respectivamente (media $=13.74$ y 14.29 , desviación estándar $=1.48$ y 2.46; rango de edad de 10 a 19 años) y nivel socioeconómico según el ingreso anual aproximado (22.1\% con ingresos de aprox. $36.153 €$; un $27.4 \%$ con ingresos de $19.933 €$; un $23.5 \%$ con ingresos de $12.902 .6 €$; un $10.6 \%$ unos 7.657.2 €). En cuanto a la situación familiar los porcentajes según la convivencia de sus miembros se distribuyó de la siguiente forma: $73 \%$, madre, padre e hijo convivían en el mismo domicilio; $17.7 \%$ padre y madre-hijo vivían en domicilios separados, en los restantes casos $(9.3 \%)$ la convivencia con las figuras maternas y paternas se alternaba por tiempos variables.

El grupo clínico se compone de un grupo incidental de niños y jóvenes derivados a tratamiento debido a las siguientes alteraciones: problemas emocionales de ansiedad y depresión ( $n=69 ; 30.5 \%)$, problemas de conducta $(n=39 ; 17.3 \%)$ y problemas de hiperactividad $(n=5$; $2.2 \%$ ). En el momento del estudio los participantes se encontraban al inicio de un tratamiento psicológico y fueron seleccionados por su terapeuta en las unidades de atención psicológica infantojuvenil a la que asistían.

Tomando este grupo como referencia se extrajo el grupo de comparación procedente de una muestra aleatoria ( $n$ =1036) extraída de la población general escolarizada.

\section{Instrumentos de evaluación}

Cuestionario de Aceptación-Rechazo parental (versión padre y madre para niños) (Child-PARQ; Rohner, 2005; Adaptación española de Del Barrio, Ramírez-Uclés, Romero y Carrasco, 2014). Se trata de un instrumento autoinformado que evalúa la aceptación y el rechazo que los hijos perciben de los padres. Consta de 29 ítems, cada uno de ellos con una escala Likert de 4 puntos: "casi siempre verdad" (4); "muchas veces verdad" (3); "a veces verdad" (2); y "casi nunca verdad" (1). La escala se compone de 4 dimensiones: 1) Rechazo (grado en el que la figura parental es percibida como poco afectuosa, fría o poco cariñosa; alfa de Cronbach = 0.84; p.ej., "No dice cosas buenas sobre mi"); 2) Hostilidad-agresión (grado en el que la figura parental es percibida como tendente al enojo, al enfado, al resentimiento y al uso de comportamientos dañinos física y verbalmente; alfa de Cronbach $=0.74$; p.ej., "Me pega, aun cuando no me lo merezco"); 3) Indiferencia-negligencia (grado en el que la figura parental es percibida como con falta de interés, desatenta o despreocupada de sus necesidades; alfa de Cronbach $=0.77$; p.ej., "No me presta atención cuando le pido ayuda"); y 4) Rechazo indiferenciado (grado en el que se perciben actitudes de rechazo en la figura parental a pesar de que no identifican comportamientos agresivos, fríos o de abandono objetivos o abiertamente identificables. Se refiere a una percepción exclusivamente fundamentada en los subjetivo; alfa de Cronbach = 0.72; p.ej., "Cuando me comporto mal me 
hace sentir que no soy querido/a"). Los alfas de Cronbach anteriores se obtuvieron en la muestra de estudio.

Se emplearon dos versiones, la versión abreviada para padres (PARQ-P) y la versión para madres (PARQ-M). Ambas son idénticas excepto en su formulación en la que cambia la figura parental a la que se refiere.

Escala de Implicación Paterna (FIS; Finley y Schwartz, 2004, adaptación española de González-Calderón, Rodríguez y Suárez, 2014). Evalua la implicación del padre ("Cuánto se implica tu padre en...") en diferentes áreas de la vida del hijo/a (e.j., social, moral, intelectual, ocio...). Consta de un total de 20 áreas cada una de las cuales se acompaña de una escala tipo Liker graduada en cuatro niveles de frecuencia desde 1 ("casi nunca") hasta 4 ("casi siempre"). Consta de dos subescalas: Implicación instrumental (se refiere a la implicación del padre en áreas relacionadas con el aprendizaje de habilidades o contenidos que sirven a los hijos como herramientas funcionales para manejarse en su vida cotidiana tales como la disciplina, la protección, el desarrollo académico, profesional y moral, los ingresos y el fomento de la independencia y la responsabilidad; alfa de Cronbach $=0.88$ ); e Implicación emocional (implicación paterna en dominios relativos al desarrollo y la seguridad emocional del hijo tales como el cuidado, el compañerismo, el ocio (diversión/jugar), actividades de intercambio/intereses, así como el desarrollo físico, emocional, social y espiritual; alfa de Cronbach = 0.78). El coeficiente alfa de Cronbach para la suma total de la escala fue de .91. Los alfas de Cronbach anteriores se obtuvieron en la muestra de estudio.

En su adaptación de ambos instrumentos a la población española, se reproducen los modelos teóricos propuestos, y presentan adecuados índices de fiabilidad y de validez de criterio (Del Barrio, Ramírez-Uclés, Romero y Carrasco, 2014; González-Calderón, Rodríguez y Suárez, 2014).

\section{Procedimiento}

Antes de iniciar el estudio se solicitó consentimiento informado a los padres y autorización a los responsables legales de los menores y a los centros clínicos y escolares a los que los niños acudían según la población de la que procedencia. En todos los casos la participación fue voluntaria y se garantizó la confidencialidad de los datos. Los cuestionarios se cumplimentaron en los centros escolares en el transcurso de una clase en horario escolar, en los casos pertenecientes al grupo general; y en las unidades de atención psicológica, en los casos pertenecientes al grupo clínico durante una sesión de evaluación individual. Una vez obtenidos los datos se procedió al análisis de los resultados. El estudio se realizó aplicando la legislación vigente sobre el tratamiento de datos confidenciales y la aprobación del Comité Bioético de la universidad en la que tuvo lugar esta investigación.

\section{Análisis estadísticos}

Se realizaron análisis descriptivos básicos, diferencias de medias, así como un análisis multivariado de la varianza (MANOVA) para determinar la influencia del estatus clínico y el sexo sobre las variables estudiadas. Además se ejecutó un análisis discriminante para determinar cuáles de las variables estudiadas pronostican mejor el estatus clínico del sujeto. Este análisis se realizó en la muestra total y en cada uno de los sexos. Para el contraste se utilizó el SPPS 15.

\section{Resultados}

\section{Diferencias por grupo y sexo en las variables} de estudio

El estudio de las diferencias por sexo y grupo se llevó a cabo mediante tres análisis multivariados de la varianza (MANOVA), uno para las variables relativas a la implicación paterna (FIS), otra para la aceptación-rechazo materna (PARQ-M) y otra para la aceptación-rechazo paterna (PARQ-P). Las medias y desviaciones tipo y los contrastes univariados se recogen en la tabla 1. Además se incluyen las medias y desviaciones típicas de la adaptación española.

En relación a la implicación paterna, los efectos multivariados para cada una de las variables no resultaron significativos ni para el sexo, ni el grupo ni su interacción: sexo (Wilks' Lambda $=0.999, F(2,221)=0.127, p=$ $0.880, \eta_{p}^{2}=0.001$ ), grupo (Wilks' Lambda $=0.987, F(2$, $\left.221)=1.437, p=0.240, \eta_{p}^{2}=0.013\right)$ y en la interacción Sexo x Grupo (Wilks' Lambda $=0.996, F(2,221)=0.449$, $\left.p=0.639, \eta_{p}^{2}=0.004\right)$. Por tanto, los niveles de implicación expresiva e instrumental del padre resultaron similares tanto entre chicos y chicas como entre el grupo clínico y general. Es decir, no aparecen diferencias en la implicación percibida del padre, ni en sus elementos expresivos ni instrumentales. Ambos grupos perciben similares niveles de implicación en uno y otro grupo

En cuanto a la aceptación-rechazo paterno, los efectos multivariados solo resultaron significativos para la variable grupo $($ Lambda de Wilks $=0.947, F(4,219)=3.058$, $\left.p=0.018, \eta_{p}^{2}=0.053\right)$ pero no para el sexo (Lambda de Wilks $\left.=0.983, F(4,219)=0.972, p=0.423, \eta_{p}^{2}=0.017\right)$ ni para la interacción Sexo x Grupo (Lambda de Wilks = $\left.0.984, F(4,219)=0.890, p=0.471, \eta_{p}^{2}=0.016\right)$. Los análisis univariados de los efectos de grupo (veasé la tabla 1) mostraron que los sujetos del grupo clínico percibían 
Tabla 1. Comparación de medias por grupo (clínico versus normativo) y sexo en las dimensiones del comportamiento parental (ANOVAs por grupo y sexo)

\begin{tabular}{|c|c|c|c|c|c|}
\hline & & Clínico & Normativo & $F$ & $\eta_{p}^{2}$ \\
\hline \multirow{3}{*}{$\begin{array}{l}\text { Implicación Expresiva padre } \\
(\text { Media }=39.34 ; D T=7.60)\end{array}$} & Chicos & $37.41(7.48)$ & $38.04(7.90)$ & .14 & .001 \\
\hline & Chicas & $36.06(7.11)$ & $38.60(7.60)$ & & \\
\hline & Total & $36.90(7.34)$ & $38.25(7.76)$ & 2.33 & .010 \\
\hline \multirow{3}{*}{$\begin{array}{l}\text { Implicación instrumental padre } \\
(\text { Media }=20.53 ; D T=3.84)\end{array}$} & Chicos & $20.77(3.86)$ & $21.40(4.34)$ & .26 & .001 \\
\hline & Chicas & $20.20(4.37)$ & $21.39(3.74)$ & & \\
\hline & Total & $20.55(4.05)$ & $21.39(4.10)$ & 2.62 & .012 \\
\hline \multirow{3}{*}{$\begin{array}{l}\text { Rechazo paterno } \\
(\text { Media }=24.54 ; D T=5.67)\end{array}$} & Chicos & $17.52(6.23)$ & $16.21(5.79)$ & .09 & .000 \\
\hline & Chicas & $18.79(5.93)$ & $15.41(4.52)$ & & \\
\hline & Total & $18.00(6.12)$ & $15.91(5.33)$ & $8.86^{*}$ & .038 \\
\hline \multirow{3}{*}{$\begin{array}{l}\text { Hostilidad paterno } \\
(\text { Media }=7.79 ; D T=2.37)\end{array}$} & Chicos & $8.77(3.42)$ & $7.87(2.40)$ & .54 & .002 \\
\hline & Chicas & $8.27(2.77)$ & $7.79(2.49)$ & & \\
\hline & Total & $8.58(3.19)$ & $7.84(2.43)$ & 3.18 & .014 \\
\hline \multirow{3}{*}{$\begin{array}{l}\text { Indiferencia paterno } \\
(\text { Media }=9.35 ; D T=2.99)\end{array}$} & Chicos & $10.25(3.32)$ & $9.67(3.10)$ & .12 & .001 \\
\hline & Chicas & $10.53(3.70)$ & $9.09(2.47)$ & & \\
\hline & Total & $10.36(3.45)$ & $9.45(2.88)$ & $5.41^{*}$ & .024 \\
\hline \multirow{3}{*}{$\begin{array}{l}\text { Rechazo indiferenciado paterno } \\
(\text { Media }=4.99 ; D T=1.85)\end{array}$} & Chicos & $5.80(2.54)$ & $5.01(1.54)$ & .44 & .002 \\
\hline & Chicas & $6.11(2.81)$ & $5.09(1.62)$ & & \\
\hline & Total & $5.92(2.64)$ & $5.04(1.56)$ & $9.21 *$ & .040 \\
\hline \multirow{3}{*}{$\begin{array}{l}\text { Rechazo materno } \\
(\text { Media }=27.13 ; D T=4.62)\end{array}$} & Chicos & $14.64(6.11)$ & $13.50(4.63)$ & .00 & .000 \\
\hline & Chicas & $15.44(5.74)$ & $12.67(4.46)$ & & \\
\hline & Total & $14.94(5.96)$ & $13.18(4.56)$ & $7.21 *$ & .031 \\
\hline \multirow{3}{*}{$\begin{array}{l}\text { Hostilidad materno } \\
(\text { Media }=7.8 ; D T=4.67)\end{array}$} & Chicos & $8.25(3.04)$ & $7.27(1.77)$ & .00 & .000 \\
\hline & Chicas & $7.97(2.42)$ & $7.53(2.06)$ & & \\
\hline & Total & $8.15(2.82)$ & $7.37(1.88)$ & $4.70^{*}$ & .021 \\
\hline \multirow{3}{*}{$\begin{array}{l}\text { Indiferencia materna } \\
(\text { Media }=8.7 ; D T=3.6)\end{array}$} & Chicos & $9.01(3.52)$ & $8.74(2.56)$ & .18 & .001 \\
\hline & Chicas & $9.60(3.53)$ & $8.51(2.32)$ & & \\
\hline & Total & $9.23(3.52)$ & $8.65(2.47)$ & 2.67 & .012 \\
\hline \multirow{3}{*}{$\begin{array}{l}\text { Rechazo indiferenciado materno } \\
(\text { Media }=4.90 ; D T=1.69)\end{array}$} & Chicos & $5.32(2.57)$ & $4.77(1.33)$ & .17 & .001 \\
\hline & Chicas & $5.39(2.16)$ & $4.48(0.98)$ & & \\
\hline & Total & $5.35(2.41)$ & $4.66(1.21)$ & $7.78^{*}$ & .034 \\
\hline
\end{tabular}

Nota. Bajo el nombre de cada variable se incluye entre paréntesis la media y desviación estándar de la adaptación española. * $p<0.05$.

significativamente mayores niveles de rechazo indiferenciado paterno, rechazo paterno e indiferencia. En cambio las variables de aceptación-rechazo paterno resultaron equiparables para chicos y chicas.

Respecto a las variables de aceptación-rechazo materno, los efectos multivariados resultaron significativos para las variables sexo (Wilks' Lambda $=0.994, F(4,219)$ $\left.=0.311, p=0.870, \eta_{p}^{2}=0.006\right)$, y grupo (Wilks' Lambda $\left.=0.947, F(4,219) \stackrel{p}{=} 3.044, p=0.018, \eta_{p}^{2}=0.053\right)$; no obstante, no resultaron significativos para la interacción Sexo x Grupo (Wilks' Lambda =0.072, F $(4,219)=1.308$, $\left.p=0.268, \eta_{p}^{2}=0.23\right)$. El análisis de los efectos univariados, mostrados en la tabla 1, indicaron que el grupo clínico informaba de niveles significativamente superiores de rechazo materno, hostilidad materna y rechazo indiferenciado. De manera similar a cómo ocurría con las variables de aceptación-rechazo paterno, en los análisis univariados las diferencias por sexo no resultaron significativas y por 
tanto chicos y chicas percibían niveles similares de implicación paterna y aceptación-rechazo parental.

\section{Análisis discriminante de los grupos a partir de las variables de estudio}

Con el fin de construir un modelo predictivo y descriptivo de la pertenencia al grupo con base en las características observadas para cada individuo, se realizaron tres análisis discriminantes uno sobre la muestra total y uno para cada uno de los sexos (véase la tabla 2).

En el discriminante realizado sobre el conjunto de la muestra, aunque el autovalor obtenido (0.09) y la correlación canónica (0.28) resultaron moderadas y los grupos mostraron un importante solapamiento (Lambda de Wilks $=0.916$ ) la diferencia entre los grupos a partir de las variables consideradas fue significativa $\left(\chi^{2}=19.135\right.$, g.l. $=$ $10, p=0.039)$. La función discriminante consiguió explicar el $8.35 \%$ de la variación de las puntuaciones de los factores de implicación paterna y aceptación-rechazo parental entre los grupos clínico y normativo. A partir de estas variables se consigue clasificar correctamente en uno u otro grupo hasta el $61.5 \%$ de los casos.
Los coeficientes de estructura de la función canónica discriminante intergrupal reflejaron que las variables que mejor diferencian los grupos y por tanto lo predicen son el rechazo indiferenciado paterno (0.67) y el rechazo paterno (0.60), seguido del rechazo indiferenciado materno (0.60), el rechazo materno $(0.55)$ y la hostilidad materna (0.54). Por tanto, la percepción de rechazo tanto materno como paterno de los jóvenes es lo que permite predecir su pertenencia al grupo clínico. Destacó el rechazo indiferenciado y el rechazo materno y paterno junto con la hostilidad exclusivamente materna. No obstante, las variables de rechazo paterno tendían a mostrar coeficientes más elevados frente a los maternos en la función discriminante.

Atendiendo a los coeficientes estandarizados las variables que mostraron una mayor asociación con el carácter clínico o no del grupo fueron la indiferencia materna $(-0.64)$ y el rechazo indiferenciado del padre $(0.59)$, en menor medida, el rechazo materno $(0.52)$ y el rechazo indiferenciado de la madre $(0.47)$

Cuando se analiza la función discriminante de los grupos por sexo, el contraste multivariado no resultó significativo ni para los chicos (Lambda de Wilks $=0.905$,

Tabla 2. Resumen de las funciones de análisis discriminante: diferenciación entre sexos y grupos

\begin{tabular}{|c|c|c|c|c|c|c|}
\hline & \multicolumn{2}{|c|}{ Chicos (140; C:70; N:70) } & \multicolumn{2}{|c|}{ Chicas (86; C:43; N:43) } & \multicolumn{2}{|c|}{ Total (226; C:113; N:113) } \\
\hline & $\begin{array}{c}\text { Coeficiente } \\
\text { Estandarizado }^{\mathrm{a}}\end{array}$ & $\begin{array}{l}\text { Coeficiente } \\
\text { Estructura }^{\mathrm{b}}\end{array}$ & $\begin{array}{l}\text { Coeficiente. } \\
\text { Estandarizado }^{\mathrm{a}}\end{array}$ & $\begin{array}{l}\text { Coeficiente } \\
\text { Estructura }^{\mathrm{b}}\end{array}$ & $\begin{array}{c}\text { Coeficiente } \\
\text { Estandarizado }^{\mathrm{a}}\end{array}$ & $\begin{array}{l}\text { Coeficiente } \\
\text { Estructura }^{\mathrm{b}}\end{array}$ \\
\hline Implicación Expresiva & .564 & -.127 & -.069 & -.380 & .282 & -.298 \\
\hline Implicación instrumental & -.487 & -.238 & .103 & -.321 & -.303 & -.343 \\
\hline Rechazo paterno & .274 & .339 & .742 & .705 & .273 & .607 \\
\hline Hostilidad paterna & -.181 & .473 & -.474 & .204 & -.159 & .436 \\
\hline Indiferencia paterna & -.068 & .283 & .003 & .508 & -.017 & .477 \\
\hline Rechazo indiferenciado & .656 & .581 & .185 & .492 & .593 & .672 \\
\hline Rechazo materno & .423 & .328 & .211 & .594 & .520 & .552 \\
\hline Hostilidad materna & .915 & .615 & -.278 & .216 & .239 & .540 \\
\hline Indiferencia materna & -.951 & .137 & -.049 & .404 & -.643 & .320 \\
\hline Rechazo indiferenciado & .038 & .423 & .744 & .595 & .471 & .601 \\
\hline Autovalor & \multicolumn{2}{|c|}{$.105^{\mathrm{a}}$} & \multicolumn{2}{|c|}{$.210^{\mathrm{a}}$} & \multicolumn{2}{|c|}{$.091^{\mathrm{a}}$} \\
\hline Wilks' Lambda (Chi cuadrado; Sig.) & \multicolumn{2}{|c|}{$.905\left(\chi^{2}=13.297 ; .208\right)$} & \multicolumn{2}{|c|}{$.826\left(\chi^{2}=15.087 ; .129\right)$} & \multicolumn{2}{|c|}{$.916\left(\chi^{2}=19.135 ; .039\right)$} \\
\hline Correlación canónica (\% explicado) & \multicolumn{2}{|c|}{$.308(9.48 \%)$} & \multicolumn{2}{|c|}{$.417(17.38 \%)$} & \multicolumn{2}{|c|}{$.289(8.35 \%)$} \\
\hline$\%$ Casos correctamente clasificados & \multicolumn{2}{|c|}{$60.7 \%$} & \multicolumn{2}{|c|}{$66.3 \%$} & \multicolumn{2}{|c|}{$61.5 \%$} \\
\hline \multirow{2}{*}{ normativo } & \multicolumn{2}{|c|}{.322} & \multicolumn{2}{|c|}{.453} & \multicolumn{2}{|c|}{.301} \\
\hline & \multicolumn{2}{|c|}{-.322} & \multicolumn{2}{|c|}{-.453} & \multicolumn{2}{|c|}{-.301} \\
\hline
\end{tabular}

Nota $.^{\mathrm{a}}=$ coeficientes y autovalores por sexo entre grupo clínico y normativo; ${ }^{\mathrm{b}}=$ Se han empleado las 1 primeras funciones discriminantes canónicas en el análisis. $\mathrm{C}=$ Clínico; $\mathrm{N}=$ Normativo. 
$\chi^{2}=13.297, p=0.208$ ), ni para las chicas (Lambda de Wilks $\left.=0.826, \chi^{2}=15.087, p=0.129\right)$, lo que indica que el conjunto de variables incluidas en la función discriminante no permite diferenciar claramente entre los grupos entre chicos y chicas por separado y por tanto, el sexo no modera el valor diferenciador de dicha función discriminante. Sin embargo esta función explica mayor porcentaje de varianza en las chicas $(17.3 \%)$ que en los chicos $(9.48 \%)$ y los pesos de cada una de las variables resultó diferente para cada uno de los sexos. Los coeficientes estandarizados que indican el peso de las variables en el efecto del grupo (clínico versus general) para cada sexo mostraron que la implicación paterna expresiva e instrumental era más robusta entre los chicos ( 0.56 y 0.48$)$ que entre las chicas $(0.06$ y 0.10$)$. Las variables de rechazo parental con mayores pesos entre los varones fueron la hostilidad materna $(0.91)$ y la indiferencia materna (-0.95), mientras que entre las chicas fueron el rechazo paterno $(0.74)$ y el rechazo indiferenciado materno (0.74).

\section{Discusión}

El objetivo del presente estudio fue analizar la capacidad predictiva y diferenciadora que el comportamiento parental percibido por los hijos tiene sobre la pertenencia a un grupo clínico o normativo de los que se derivan claras implicaciones sobre el estado psicológico de los menores. El análisis se realizó considerando elementos cuantitativos (implicación expresiva e instrumental) del comportamiento de la figura paterna y elementos cualitativos (aceptación-rechazo) de ambas figuras parentales teniendo en cuenta el sexo de los hijos.

Los resultados mostraron que el rechazo materno y paterno percibido por los hijos era significativamente superior entre los niños del grupo clínico frente al general y que estas diferencias no estaban condicionadas por el sexo de los hijos.

Estos resultados son consistentes con los trabajos que muestran la asociación entre el rechazo parental y el ajuste psicológico de los hijos (Khaleque, 2015; Khaleque y Rohner, 2012). De acuerdo con estos estudios, las relaciones parentales de amor y cariño son un requisito para satisfacer las necesidades de afecto de los hijos, facilitar la construcción de un modelo representacional del mundo como un espacio confiado y seguro e identificar a las figuras principales de referencia como fuentes de aprendizaje y socialización. No obstante, los resultados obtenidos en este trabajo contribuyen a los pocos estudios que han analizado el papel del rechazo parental en la sintomatología clínica y la potencial pertenencia de los hijos a un grupo clínico que requiere atención psicológica.
El tipo de rechazo más predictivo en la discriminación entre grupos fue la de rechazo indiferenciado en ambas figuras parentales. El rechazo indiferenciado se refiere a los sentimientos que experimentan los hijos de no ser queridos o ser despreciados sin tener necesariamente ningún indicador explícito u objetivo de rechazo. Frente a las demás dimensiones de aceptación-rechazo, el rechazo indiferenciado se caracteriza por su mayor carga subjetiva, la cual trasciende a los indicadores observables sobre los que se les pregunta a los niños. Si bien no hemos encontrado trabajos que informen sobre este particular y para el que no tenemos una explicación definitiva, estos datos sugieren la inevitable relación entre los aspectos perceptivos y clínicos que desde la psicología cognitiva se ha enfatizado (Beck, 1976; Ellis, 1979a, 1979b). Es posible que el carácter clínico y sintomatológico de los niños pueda condicionar la percepción de los padres más allá del comportamiento real de éstos y sea el propio malestar de los hijos el que les hace percibir sesgadamente este comportamiento de rechazo en los padres (Gotlib y Joormann, 2010). En este sentido, es importante recordar el valor percibido de estas medidas y la necesidad de atender a la observación directa de indicadores para conocer el verdadero sentido de estas asociaciones. Más allá de este posible sesgo en la percepción de los hijos, no podemos obviar que las otras dimensiones de rechazo percibidas que también resultaron significativas (p.ej.,rechazo, hostilidad) contenían indicadores observables sobre los que los hijos también informaron (p.ej., "Hace todo lo posible por herir mis sentimientos", "No me trata amablemente y con cariño").

Aunque el rechazo y el rechazo indiferenciado fueron dimensiones compartidas por ambas figuras parentales en la predicción de la pertenencia de los hijos a uno u otro grupo, si atendemos a las diferencias entre las dimensiones paternas y maternas de aceptación-rechazo percibidas por los hijos, los resultados revelan que el patrón de rechazo materno asociado al grupo clínico incluye elementos de hostilidad y el del padre, elementos de indiferencia/ negligencia. Los hijos pertenecientes al grupo clínico percibían mayor hostilidad (p.ej.,ira, resentimiento, agresión) en las madres y mayor indiferencia/negligencia en los padres. Puede que la dedicación en las tareas más cotidianas y el mayor tiempo que las madres tienden a pasar con los hijos (Bornstein, 2013) explique estas diferencias: por una parte la mayor presencia de conflictividad con la figura materna y la percepción de ausencia o abandono de la figura paterna. Hay evidencia que ha mostrado diferencias en la calidad de la relación padres-hijos y madres-hijos por ejemplo el nivel de aceptación, la frecuencia y tipo de conflictos (Dwairy, 2010) así como sobre los efectos diferenciales de cada una de estas figuras sobre distintos indicadores de ajuste psicológico en los hijos (Putnick et al., 
2014). No obstante, el número de estudios sobre relaciones familiares que evalúan efectos de la relación con cada uno de los padres es aún limitado.

Los resultados además mostraron que la contribución predictiva de los padres sobre la pertenencia de los hijos a uno u otro grupo tendía a ser mayor que la de las madres. Estos resultados aunque no siempre se constatan en la bibliografía (Miranda, Affuso, Esposito, y Bacchini, 2016) son consistentes con un metanálisis reciente (Khaleque y Rohner, 2012) en el que encuentran que el ajuste psicológico de los hijos tiende a ser mejor explicado por la aceptación-rechazo paterno que materno. Es complicado encontrar una explicación a estos resultados, especialmente en una cultura como la nuestra en la que la madre aún asume el principal rol de cuidadora y educadora en el contexto familiar. Tal vez y como mera especulación que debería ser empíricamente explorada en el futuro, la intensidad del rechazo paterno frente al materno podría producir un efecto sinérgico o sumativo al rechazo de la madre que marcara la diferencia para predecir la potencial derivación de un niño a un servicio clínico. En cualquier caso, lo importante no parece ser el sexo de los padres sino las relaciones padre/madre-hijos lo que mejor predice la condición clínica de éstos.

Otro elemento importante a destacar en los presentes resultados es la prominencia de los elementos cualitativos de la relación padres-hijos frente a los cuantitativos. La implicación paterna no resultó un predictor significativo ni relevante en la pertenencia de los hijos a uno u otro grupo. Esta diferencia claramente se ha constatado sobre la figura paterna en los estudios iniciales desarrollados (Lamb, 1997; Pleck, 2010) que tradicionalmente han analizado los componentes más cuantitativos. En línea con la bibliografía revisada, se constata que los elementos cualitativos como la calidez paternal y el apoyo son más importantes para el ajuste de los niños, que elementos cuantitativos como el tiempo que los padres pasan cuidando de los hijos o la mera participación en sus actividades (Carrasco, Holgado-Tello y Rodríguez-Serrano, 2015; Fagan, Day, Lamb y Cabrera, 2014; Malmberg y Flouri, 2011).

En relación con el sexo de los hijos, los resultados no hallaron diferencias ni en la predicción al grupo de pertenencia ni en el comportamiento parental. Estos resultados fueron consistentes con el metanálisis de Ali et al., (2015) en el que no aparecieron diferencias pero contradicen aquellos otros trabajos que encontraron un comportamiento parental diferencial entre hijos e hijas ( Ali y Zubair, 2011).

Finalmente, y aunque los datos presentados en este trabajo aportan nueva evidencia sobre el papel del comportamiento parental en el ajuste de los hijos y su poten- cial pertenencia a un grupo de significación clínica, es necesario considerar algunas limitaciones. En primer lugar y desde una perspectiva más sistémica del contexto familiar (Minuchin, 2016) el estudio propuesto no incorpora ni la totalidad de sistemas (p.ej., hermanos, relación padre-madre, etc.), ni las diferentes tipologías de estructura familiar (p.ej., divorcio, adopción, monoparentalidad), ni el conjunto posible de relaciones bidireccionales que potencialmente ocurren en las dinámicas interpersonales, particularmente en la familia (Bronfenbrenner, 2009; Sameroff, 2009). En este sentido, el presente trabajo analiza las relaciones desde un diseño unidireccional en el que se analizan los efectos de los padres sobre los hijos y no al revés. Abundante bibliografía reconoce el efecto de los hijos como un elemento fundamental que puede explicar el comportamiento parental y por ende el propio ajuste de los hijos (Goodman, 2014; Meunier, Roskam, y Browne, 2011). Este efecto de los hijos incluye desde sus características temperamentales, su capacidad de afrontamiento, hasta su estado de salud o condición clínica (Gotlib y Joormann, 2010; Gross, Shaw, Burwell y Nagin, 2009;). En segundo lugar, las medidas utilizadas son autoinformes y refieren experiencias de rechazo percibidas. La medida del rechazo mediante instrumentos de observación podría encontrar resultados diferentes. En tercer lugar, la muestra clínicas es incidental lo que limita la generalización de los resultados a otros grupos clínicos. La investigación futura se beneficiaría de una evaluación más amplia del funcionamiento de la familia, incluyendo los aspectos y variables anteriormente citados. A pesar de estas limitaciones, consideramos que los resultados obtenidos son un primer paso para profundizar en las dinámicas familiares relacionadas con el rechazo parental que están asociadas al desajuste psicológico de los hijos y que pueden llevar a éstos a necesitar una atención psicológica especializada.

En conclusión, el estudio desarrollado muestra el papel de la aceptación parental percibida por los hijos como un factor protector para el ajuste saludable. En este sentido la negligencia y el rechazo de los padres incrementan la probabilidad de los hijos a manifestar una alteración clínica. Por tanto, la promoción del comportamiento parental que promueva el afecto y cariño hacia los hijos debería ser contemplada como objetivo en los programas para la prevención de los problemas psicológicos en los hijos.

\section{Referencias}

Ali, M., \& Zubair, A. (2011). Parental Attachment, Parental Acceptance, and Aggression among Adolescents. Pakistan Journal of Psychology, 42 (2). 
Ali, S., Khaleque, A., \& Rohner, R. P. (2015). Pancultural gender differences in the relation between perceived parental acceptance and psychological adjustment of children and adult offspring: A meta-analytic review of worldwide research. Journal of Cross-Cultural Psychology, 46, 1059-1080. doi: 10.1177/ 0022022115597754

Asbourne, L., Daly, K. J., \& Brown, J. L. (2011). Responsiveness in father-child relationships: The experience of fathers. Fathering, 9, 69-86. doi: 10.3149/fth.0901.69.

Beck, A. T. (1976). Cognitive therapy and the emotional disorders. New York: Universities Press.

Bisht, J., Sankhyan, N., Kaushal, R. K., Sharma, R. C., \& Grover, N. (2008). Clinical profile of pediatric somatoform disorders. Indian pediatrics, 45, 111. doi: 10.1007/s12098-010-0282-z

Bornstein, M. H. (2013). Parenting and child mental health: A cross-cultural perspective. World Psychiatry, 12 (3), 258-265.

Bronfenbrenner, U., \& Bronfenbrenner, U. (2009). The ecology of human development: Experiments by nature and design. Harvard university press.

Carrasco, M. A., Holgado, F. P., Rodríguez, M. A., \& del Barrio, M. V. (2009). Concurrent and across-time relations between mother/father hostility and children's aggression: A longitudinal study. Journal of Family Violence, 24, 213-220. doi 10.1007/s10896-009-9222-y

Carrasco, M. Á., Holgado-Tello, F. P., \& Rodríguez-Serrano, M. Á. (2015). Intraparental Inconsistency: The Influence of Parenting Behaviors on Aggression and Depression in Children. Family Relations, 64, 621-634. doi: 10.1111/fare.12168

Del Barrio, V. D., Ramírez-Uclés, I., Romero, C., y Carrasco, M. Á. (2014). Adaptación del Child-PA RQ/Control: Versiones para el padre y la madre en población infantil y adolescente española. Revista de Acción Psicológica, 11, 27-46. doi: 10.5944/ap.11.2.1417

Demidenko, N., Manion, I., \& Lee, C. M. (2015). Father-Daughter Attachment and Communication in Depressed and Nondepressed Adolescent Girls. Journal of Child and Family Studies, 24 (6), 1727-1734. doi: 10.1007/s10826-014-9976-6.

Dwairy, M. (2010). Parental acceptance-rejection: A fourth cross-cultural research on parenting and psychological adjustment of children. Journal of Child and Family Studies, 19, 30-35. doi: 10.1007/s10826-009-9338-y

Ellis, A. (1979a). The theory of rational-emotive therapy. En A. Ellis y J. M. Whiteley (Eds.), Theoretical and empirical foundations of rational-emotive therapy (pp. 33-60). Monterrey, CA: Brooks/Cole.

Ellis, A. (1979b). The practice of rational-emotive therapy. En A. Ellis y J. M. Whiteley (Eds.), Theoretical and empirical foundations of rational-emotive therapy (pp. 61-100). Monterrey, CA: Brooks/Cole.

Fagan, J., Day, R., Lamb, M. E., \& Cabrera, N. J. (2014). Should Researchers Conceptualize Differently the Dimensions of Parenting for Fathers and Mothers? Journal of Family Theory $y$ Review, 6, 390-405. doi: 10.1111/jftr.12044

Finley, G. E., \& Schwartz, S. J. (2004). The Father Involvement and Nurturant Fathering Scales: Retrospective measures for adolescent and adult children. Educational and Psychological Measurement, 64, 143-164. doi: 10.1177/0013164403258453

Flouri, E., Midouhas, E., \& Narayanan, M. K. (2015). The relationship between father involvement and child problem beha- viour in intact families: a 7-year cross-lagged study. Journal of abnormal child psychology, 44, 1011-1021. doi:10.1007/ s10802-015-0077-9

González-Calderón, M. J., Rodríguez, M. Á., y Suárez, J. C. (2014). Propiedades psicométricas y dimensionalidad de la versión española para niños y adolescentes del Father Involvement Scale (FI S). Revista de Acción Psicológica, 11, 61-76. doi: 10.5944/ap.11.2.14175.

Goodman, S. N. (2014). Maternal depression in association with fathers' involvement with their infants: Spillover or compensation/buffering? Infant Mental Health Journal, 35, 495-508. doi:10.1002/imhj.21469

Gotlib, I. H., \& Joormann, J. (2010). Cognition and depression: Current status and future directions. Annual Review of Clinical Psychology, 6, 285-312. doi:10.1146/annurev.clinpsy.121208.131305.

Gross, H. E., Shaw, D. S., Burwell, R. A., \& Nagin, D. S. (2009). Transactional processes in child disruptive behavior and maternal depression: A longitudinal study from early childhood to adolescence. Development and Psychopathology, 21, 139156. doi: 10.1017/S0954579409000091

Hale II I, W. W., VanderValk, I., Akse, J., \& Meeus, W. (2008). The interplay of early adolescents' depressive symptoms, aggression and perceived parental rejection: A four-year community study. Journal of Youth and Adolescence, 37 (8), 928-940. doi: 10.1007/s10964-008-9280-0

Khaleque, A. (2015). Perceived parental neglect, and children's psychological maladjustment, and negative personality dispositions: A meta-analysis of multi-cultural studies. Journal of Child and Family Studies, 24, 1419-1428.doi: 10.1007/ s10826-014-9948-x

Khaleque, A., \& Rohner, R. P. (2012). Pancultural Associations Between Perceived Parental Acceptance and Psychological Adjustment of Children and Adults A Meta-Analytic Review of Worldwide Research. Journal of Cross-Cultural Psychology, 43, 784-800. doi: 10.1177/0022022111406120

Kostic, J. S., Nešić, M., Stankovic, M., \& Žikić, O. (2014). Perceived parental acceptance/rejection, some family characteristics and conduct disorder in adolescents. Vojnosanitetski pregled, 71 (10), 942-948.

Kwon, K.-A., Jeon, H.-J., Lewsader, J. T., \& Elicker, J. (2012). Mothers' and fathers' parenting quality and toddlers' interactive behaviors in dyadic and triadic family contexts. Infant and Child Development, 21, 356-373. doi: 10.1002/icd.1746

Lamb, M. (1997). Father and child development: An introductory overview and guide. En M. E. Lamb (Ed.), The role of the father in child development (pp. 73-133). New York: Wiley

Lamb, M. E. (2012). Mothers, fathers, families, and circumstances: Factors affecting children's adjustment. Applied Developmental Science, 16, 98-111. doi: 10.1080/10888691.2012.667344

Malik, F. (2012). Parental acceptance-rejection and paternal authoritarianism among abused children in Pakistan. Journal of Behavioural Sciences, 22, 23-48.

Malmberg, L- E., \& Flouri, E. (2011). The comparison and interdependence of maternal and pater nal influences on young children's behavior and resilience. Journal of clinical child y adolescent psychology, 40 (3), 434-444. doi: 10.1080/15374416. 2011.563469

Meunier, J. C., Roskam, I., \& Browne, D. T. (2011). Relations between parenting and child's behavior: Exploring child's per- 
sonality and parental self-efficacy as third variables. International Journal of Behavioral Development, 35, 246-259. doi: 10.1177/0165025410382950.

Minuchin, S. (2016). L'arte della terapia familiare. Rivista di psicoterapia relazionale, 43, 5-13.

Miranda, M. C., Affuso, G., Esposito, C., \& Bacchini, D. (2016). Parental Acceptance-Rejection and Adolescent Maladjustment: Mothers' and Fathers' Combined Roles. Journal of Child and Family Studies, 25 (4), 1352-1362. doi:10.1007/ s10826-015-0305-5

Najam, N., \& Kausar, R. (2012). Father Acceptance-Rejection, Father Involvement and Socio Emotional Adjustment of Adolescents in Pakistan. Journal of Behavioural Sciences, 22 (1), $1-22$.

Pleck, J. H. (2010). Paternal involvement: Revised conceptualization and theoretical linkages with child outcomes. The role of the father in child development (5th ed., pp. 5893). Hoboken, NJ: John Wiley y Sons

Putnick, D. L., Bornstein, M. H., Lansford, J. E., Malone, P. S., Pastorelli, C., Skinner, A. T., ..., \& Alampay, L. P. (2014). Perceived mother and father acceptance-rejection predict four unique aspects of child adjustment across nine countries. Journal of Child Psychology and Psychiatry, 56 , 923-932. doi: 10.1111 jcpp. 12366
Rohner, R. P. (2005). Parental acceptance-rejection questionnaire (PA RQ): Test manual. In R. P. Rohner y A. Khaleque (Eds.), Handbook for the study of parental acceptance and rejection, 4th ed. (pp. 43-106). Storrs, CT: Rohner Research Publications Rohner, R. P. (2014). The Parental "Acceptance-Rejection Syndrome": Universal Correlates of Perceived Rejection. American Psychologist, 59, 830-840. doi: 10.1037/0003-066X.59.8.830.

Rohner, R. P., \& Khaleque, A. (2005). Personality Assessment Questionnaire (PA Q): Test manual. En R. P. Rohner y A. Khaleque (Eds), Handbook for the study of parental acceptance and rejection ( $4 .^{\mathrm{a}}$ ed) (pp 187-225). Storrs. CT: Rohner Research Pub.

Rohner, R. P., Khaleque, A., \& Cournoyer, D. E. (2012). Parental acceptance-rejection theory, methods, evidence and implications. Retrieved from http://csiar.uconn.edu/wp-content/ uploads/sites/494/2014/02/IN TROD UCTI ON-TO PARE NTAL- ACCE PTAN CE-3-27-12.pdf

Sameroff, A. (2009). The transactional model of development. How children and contexts shape each other. Washington, DC: American Psychological Association. doi: 10.1037/11877-000

Xing, Z., Hou, X., Zhou, K., Qin, D., \& Pan, W. (2014). The impact of parental-rearing styles on irritable bowel syndrome in adolescents: A school-based study. Journal of Gastroenterology and Hepatology, 29, 463-468. doi: 10.1111/jgh.12388 